\title{
The risk of exaggerated risk aversion-a life and death struggle for molecular imaging
}

\author{
Bengt Långström • Anders Grahnen • Per Hartvig Honoré • Jürgen Borlak • \\ Mats Bergstrom • Bengt Nielsen • JeanLuc Vanderheyden • Yasuyoshi Watanabe • \\ Raymond Josephsson • Poul F. Høilund-Carlsen • Markus Schwaiger • \\ Steven M. Larson • David M. Goldenberg • Andreas Melzer • Henry Engler • \\ Rodney Hicks • Anders Sundin • Marko Seppänen • Göran Hedenstierna • \\ Agneta Nordberg • David Brooks
}

Published online: 18 July 2009

(C) The Author(s) 2009. This article is published with open access at Springerlink.com

\section{Dear Sir,}

In a famous Swedish cartoon from the mid-1980s (Staffans Stollar), two men have a discussion in which one states: "Life is so dangerous that it is strange that the National Board of Health \& Welfare allows it!" This cartoon encapsulates our perceptions of the risk-benefit analysis of certain regulations in experimental medicine, particularly as they pertain to the development of new molecular imaging tracers.

\section{B. Långström $(\bowtie)$}

Department of Biochemistry and Organic Chemistry,

Uppsala University,

Uppsala, Sweden

e-mail: Bengt.Langstrom@biorg.uu.se

\section{A. Grahnen}

Quintiles AB, Uppsala University,

Uppsala, Sweden

\section{P. H. Honoré}

Department of Pharmacology and Pharmacotherapy,

University of Copenhagen,

Copenhagen, Denmark

\section{J. Borlak}

Pharmaco- and Toxicogenomics,

Fraunhofer Institute for Toxicology and Experimental Medicine,

Medical School of Hannover,

Hannover, Germany

\section{Bergstrom $\cdot$ R. Josephsson}

Clinical Imaging Platform, F. Hoffmann-La Roche,

Basel, Switzerland

B. Nielsen

Academics Program, GE Healthcare International,

Stockholm, Sweden
From a social point of view, risk aversion is a prominent human trait, but one which is not always rational or guided by statistical support. Today, in medical science, we demand an evidence-based approach that is both wellinformed and logical. So how do we handle and interpret absolute risks in exploratory medical research and how should we compare it with other risks? There is a strong tendency to exaggerate immediate and acute risks and downplay the associated potential long-term benefits.

J. Vanderheyden

Global Molecular Imaging, GE Healthcare,

Waukesha, WI, USA

Y. Watanabe

RIKEN Centre of Molecular Imaging Science,

Kobe, Japan

R. Josephsson

Medical Sciences Department,

Uppsala University,

Uppsala, Sweden

P. F. Høilund-Carlsen

Department of Nuclear Medicine,

Odense University Hospital,

Odense, Denmark

M. Schwaiger

Nuklearmedizinische Klinik und Poliklinik,

Klinikum rechts der Isar der Technischen Universität München,

Munich, Germany

S. M. Larson

Nuclear Medicine Service, Gerschel PET Center,

Ludwig Institute for Cancer Immunotherapy,

Memorial Sloan-Kettering Cancer Center,

New York, NY, USA 
Although the FDA in their ground-breaking "Critical Path Initiative" points to the need for more exploratory studies in humans, the existing dogma followed by most regulatory bodies is to avoid all potential risks if benefits are difficult to assess in the short term. This may seem to be a wise strategy; however, it is perceived by many of us as leading to stagnation in clinical research and missed opportunities to gain new knowledge in the complex biology of man. Benefits are not always easy to predict. However, if the foreseeable risk is small (with a certain probability), potential benefits must be put into the equation! Current PET technology utilizes radionuclides in minute (homeopathic) doses, but with high specific radioactivity. It is actually implicit in the broad acceptance of the "microdosing" concept advocated for the development of therapeutic agents that such tiny chemical quantities are likely to be safe.

Moreover, the radiation exposure is low, generally being comparable to that obtained from 1 year of natural background radiation. Despite these considerations, a new PET tracer being used in early clinical development and planned for use in perhaps only a handful of patients is, from a regulatory perspective, viewed as a new drug in many parts of the world. In Europe, in particular, new PET tracers are required to undergo full GMP qualification of its production. While new "microdosing" guidelines have simplified toxicological evaluation and represent a significant step forward, European guidelines still require traditional genotoxicity tests, although the human exposure to a PET tracer might be many 1,000 -fold lower than that from traditional drugs. Almost the same requirements are put on the production of a PET tracer that will be tested in only a few individuals as on the production of new therapeutic drugs that will potentially be used in longterm clinical trials involving thousands of patients. Clearly, the risks to society are dramatically different!

We care deeply about progress in medicine and are vitally concerned for the safety of our current volunteers and patients, while at the same time trusting that novel scientific knowledge can bring benefits to future patients. Therefore, we argue that there is an urgent need for a joint effort to reduce hurdles to radiotracer development and provide a better balance between the regulatory actions aimed at diminishing risk and fostering early human studies that can benefit science. Our intention as scientists in the medical field is never to harm but to strive to help and to support society by combating disease. In this we do not need hindrance, but support from public and private regulatory bodies. Let's do this together!

We end this note with a slightly modified quotation by Albert Einstein: "The world is dangerous to live in, not because of those who do evil (hinder), but because of those who look on and let them do so."

Uppsala, 7 April 2009

Open Access This article is distributed under the terms of the Creative Commons Attribution Noncommercial License which permits any noncommercial use, distribution, and reproduction in any medium, provided the original author(s) and source are credited.

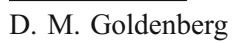

Garden State Cancer Center, Belleville, Immunomedics, Inc., Morris Plains, NJ, USA

\author{
A. Melzer \\ Dundee \& St. Andrews \& Ninewells Hospital, \\ University Wilson House, \\ Dundee, UK \\ H. Engler \\ CUDIM, \\ Montevideo, Uruguay \\ R. Hicks \\ Departments of Medicine and Radiology, \\ Centre for Molecular Imaging, University of Melbourne, \\ Melbourne, Australia \\ R. Hicks \\ Translational Research Group, \\ The Peter MacCallum Cancer Centre, \\ Melbourne, Australia

\author{
A. Sundin \\ Department of Radiology, Karolinska University Hospital, \\ Stockholm, Sweden \\ M. Seppänen \\ Department of Nuclear Medicine, Turku University Hospital, \\ Turku, Finland \\ G. Hedenstierna \\ Department of Clinical Physiology, University Hospital, \\ Uppsala, Sweden \\ A. Nordberg \\ Division of Alzheimer Neurobiology, \\ Clinic of Geriatric Medicine, Karolinska University Hospital, \\ Karolinska Institutet, \\ Huddinge, Sweden \\ D. Brooks \\ Faculty of Medicine, Division of Neuroscience, \\ Imperial College, \\ London, UK
}

